\title{
An Analysis of the Potential Relationship between Crowe Type and Lower Extremity Morphology in Patients with Developmental Dysplasia of the Hip
}

\author{
Ömer Naci Ergin, MD, Serkan Bayram, MD, Fikret Berkan Anarat, MD, \\ Mehmet Ekinci, MD, Lezgin Mert, MD, Emre Özmen, MD, İrfan Öztürk, MD \\ Department of Orthopaedics and Traumatology, Faculty of Medicine, Istanbul University, Istanbul, Turkey
}

Purpose: To test whether Crowe type is related to femoral alignment and leg length discrepancy by evaluating the preoperative lengths and coronal alignment of femurs, pelvic parameters and hip morphology of patients who underwent primary hip arthroplasty due to coxarthrosis secondary to developmental dysplasia of the hip (DDH). Materials and Methods: Medical records of patients with coxarthrosis secondary to DDH who were treated with total hip arthroplasty at Department of Orthopaedics and Traumatology, Istanbul University Faculty of Medicine between 2008 and 2017 were reviewed. The mechanical axis of lower limbs was analyzed; pelvic height and femoral and tibial lengths were measured. All femurs were classified according to the Dorr classification.

Results: A total of 97 patients were eligible for analysis and were diagnosed with unilateral DDH ( $\mathrm{n}=51)$ or bilateral DDH $(n=46)$. In those diagnosed with unilateral DDH, the affected pelvis, femur, and tibia were often shorter than the unaffected side. In those diagnosed with bilateral DDH, femoral and pelvic lengths were unpredictable. In the femoral coronal alignment test, data varied widely but were within normal limits. The difference in the Dorr types of femurs was significant between dysplastic and normal sides of patients with unilateral DDH $(P=0.001)$ but not those with bilateral DDH.

Conclusion: Especially in patients with unilateral DDH, pelvic heights and femoral and tibial lengths on the affected side may be shorter compared with unaffected side regardless of the Crowe type. Femoral coronal alignment is unpredictable for both groups. Careful preoperative analyses of femoral coronal alignment and pelvic length are advised.

Key Words: Hip dislocation, congenital, Arthroplasty, replacement, hip, Leg length inequality, Lower extremity

Submitted: November 21, 2019 1st revision: December 31, 2019 2nd revision: February 1, 2020 3rd revision: February 20, 2020

Final acceptance: February 20, 2020

Address reprint request to

Serkan Bayram, MD

(https://orcid.org/0000-0001-7651-1200)

Department of Orthopaedics and Traumatology, Faculty of

Medicine, Istanbul University, Topkapi, Turgut Özal Millet Cd,

34093 Fatih/i stanbul, Turkey

TEL: +90-212-414-20-00 FAX: +90-212-410-15-00

E-mail: dr.serkanbayram89agmail.com
This is an Open Access article distributed under the terms of the Creative Commons Attribution Non-Commercial License (http://creativecommons. org/licenses/by-nc/4.0) which permits unrestricted non-commercial use, distribution, and reproduction in any medium, provided the original work is properly cited. 


\section{Hip \& Pelvis}

Hip Pelvis 32(2): 85-92, 2020

\section{INTRODUCTION}

The primary aim of total hip arthroplasty (THA) is to relieve pain and recover a functional range of motion of the hip. Deformities and differences in the length of lower extremities are also corrected in certain patients ${ }^{1)}$. After THA, leg length discrepancy can be a source of patient dissatisfaction even if the pain disappears and a functional hip is achieved. Unequal leg lengths, especially in female patients, is a major cause of dissatisfaction after a THA surgery $^{2}$. THA has also been reported as a highly successful procedure for functional recovery, leg length correction, and pain reduction in patients with coxarthrosis secondary to developmental dysplasia of the hip (DDH) ${ }^{3}$.

Particular anatomical differences are observed in patients with DDH. Femoral deformities such as increased anteversion, aspheric femoral head, and reduced femoral head-neck offset have been reported in patients with acetabular dysplasia ${ }^{4}$. Some studies also suggest that the femoral neck can be longer on the affected side in patients with unilateral dysplasia ${ }^{5}$. We hypothesized that the Crowe type DDH affects femoral alignment, femoral medullary canal, and lower extremity length. Therefore, this study aimed to test this hypothesis by evaluating the preoperative lengths and coronal alignment of femurs and pelvis of patients who underwent primary hip arthroplasty due to coxarthrosis secondary to DDH.

\section{MATERIALS AND METHODS}

\section{Ethics Statement and Subjects}

This retrospective study was conducted by evaluating the records of 211 patients with coxarthrosis secondary to DDH who were treated with THA at Department of Orthopaedics and Traumatology, Istanbul University Faculty of Medicine between 2008 and 2017 after receiving approval from the ethics committee of Istanbul University Faculty of Medicine (No. 2018/412).
Inclusion criteria required that patients were $\geq 18$ years and diagnosed with DDH in the absence of other congenital or acquired deformities of the hip joint. Patients with previous hip or knee surgery, coxarthrosis secondary to other causes (e.g., trauma, avascular femoral head necrosis, tumor, septic arthritis, and rheumatologic origin) were excluded from the study. Of these 211 patients, 97 (73 female and 24 male) were eligible: 51 with unilateral DDH and 46 with bilateral DDH. The two subcohorts were analyzed separately.

All patients had anterior-posterior (AP) pelvic radiographs and standard full-length weight-bearing lower extremity radiographs. Preoperative full-leg radiographs were obtained with patients in a bipedal stance, with knees at maximal extension, feet in neutral rotation, patellae pointing forward, and using blocks under the shorter extremity to correct the coronal pelvic tilt. Picture archiving and communication system (PACS) (Extreme PACS, Ankara, Turkey) was used

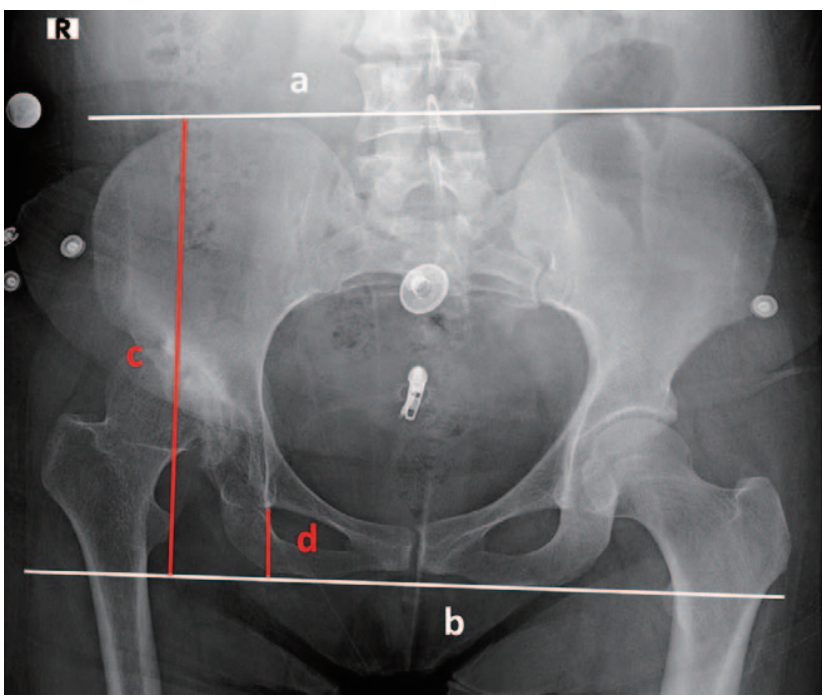

Fig. 1. a, b: pelvic orientation lines, c: the height of the hemipelvis was measured as the distance between the lines connecting the highest points of the iliac crest to the line across the lower edges of the ischial tuberosities, $d$ : ischial height was measured as the distance from the teardrop to the line across the bottom edges of bilateral ischial tuberosity.

Table 1. Description of Crowe Classification

\begin{tabular}{ll}
\hline \hline Group & \multicolumn{1}{c}{ Description } \\
\hline Group 1 & Subluxation $<50 \%$ or proximal dislocation $<0.1 \%$ of the pelvic height \\
Group 2 & Subluxation $50-75 \%$ or proximal dislocation of $0.1-0.15 \%$ of the pelvic height \\
Group 3 & Subluxation $75-100 \%$ or proximal dislocation of $0.15-0.20 \%$ of the pelvic height \\
Group 4 & Subluxation $>100 \%$ or proximal dislocation $>0.25 \%$ of the pelvic height \\
\hline
\end{tabular}




\section{Hip \& Pelvis}

Ömer Naci Ergin et al. Lower Extremity Morphology in Patients with Hip Dysplasia

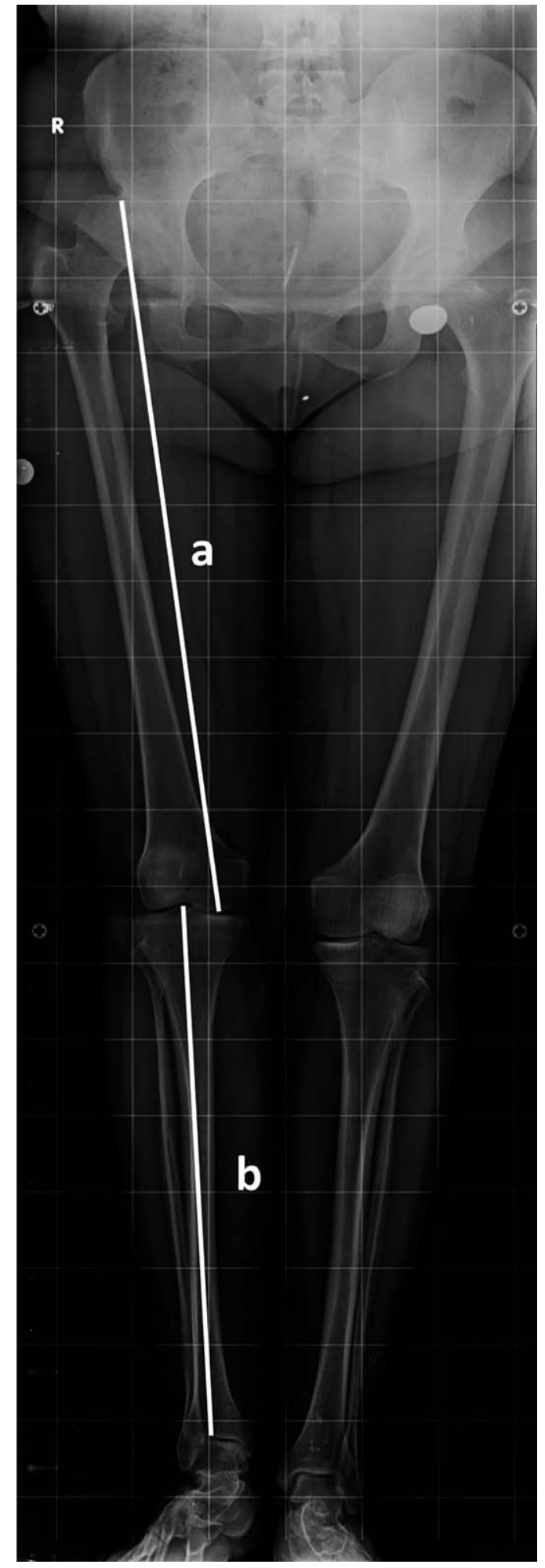

Fig. 2. a: femoral length was measured from the uppermost endpoint of the femoral head to the femoral medial condyle, b: tibial length was measured from the uppermost endpoint of the proximal tibial joint line to the ankle joint.

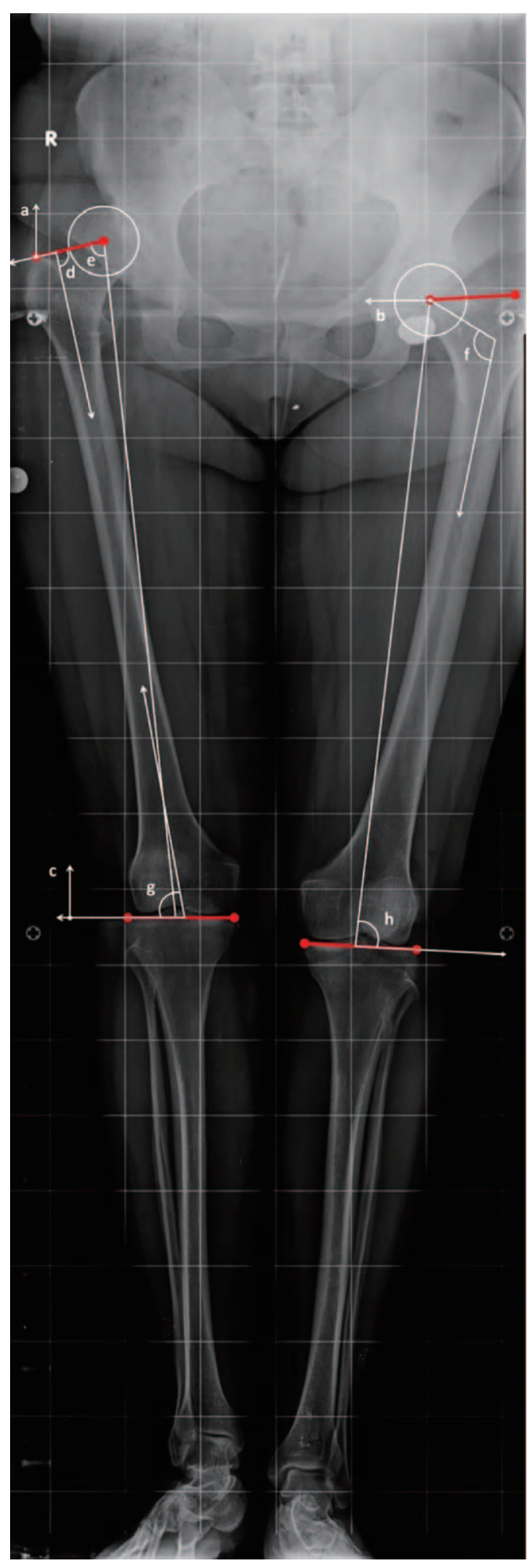

Fig. 3. a: proximal femoral orientation line, b: rotation center of hip, c: distal femoral orientation line, $d$ : anatomic-medial proximal femoral angle, e: mechanic-Lateral proximal femoral angle, f: neck-shaft angle, $g$ : anatomic-lateral distal femoral angle, h: mechanic-lateral distal femoral angle. 
for all measurements.

Hips were classified according to the Crowe system (Table 1), based on the subluxation of femoral heads from the true acetabulum, and correlated with the magnitude and anatomic deformation pattern of the dysplastic hips ${ }^{6}$.

Pelvic heights were measured as the distance between the lines connecting the highest points of the iliac crest to the line across the lower edges of the ischial tuberosities. The femoral length was measured from the uppermost endpoint of the femoral head to the femoral medial condyle. The tibial length was measured from the uppermost endpoint of the proximal tibial joint line to the ankle joint (Fig. 1, 2 ). The threshold selected for significant difference was $0.5 \mathrm{~cm}^{7)}$.

The angle formed between the joint line and either the mechanical or anatomical axis is known as the joint orientation angle. The name of each angle specifies whether it is measured based on a mechanical or an anatomic axis. The angle may be measured medial, lateral, anterior, or posterior to the axis line. The angle may refer to the proximal or distal joint orientation of the femur or tibia.

The mechanical axis of the lower limb, mechanical lateral distal femoral angle (mLDFA), anatomic lateral distal femoral angle (aLDFA), mechanical lateral proximal femoral angle (mLPFA), anatomic medial proximal femoral angle (aMPFA), and femoral medial neck shaft angle (MNSA) were analyzed in all patients (Fig. 3).

Femurs were qualitatively assessed based on three distinct shape patterns and bone structure of the proximal femur ${ }^{8)}$. Type-A femurs have thick medial and lateral cortices on the AP radiographs and a large posterior cortex on lateral radiographs. Thick diaphyseal cortices cause the funnelshaped proximal femur. Type-B femurs have bone loss from the medial cortex on AP radiographs and from the posterior cortex on lateral radiographs. Their intramedullary canals are wider than that in type-A femurs. Type- $\mathrm{C}$ femurs are those which: (i) have lost nearly all medial and posterior cortices and (ii) are thin and might display a fuzzy appearance on radiographs ${ }^{8)}$.

All measurements were taken twice by the two authors (F.B.A and M.E) independently with an interval of one week between measurements to decrease intraobserver (interand intraclass correlation coefficients $[\mathrm{ICCs}]=0.992,95 \%$ confidence interval $[\mathrm{CI}]=0.987-0.995)$ and interobserver errors $(\mathrm{ICCs}=0.994,95 \% \mathrm{CI}=0.991-0.997)$.

\section{Statistical Analyses}

Data analyses were performed using IBM SPSS ver. 22.0 (IBM Corp., Armonk, NY, USA). The normality of distribution was tested using the Shapiro-Wilk test. Pearson correlation analysis was used to evaluate the relationships between variables with normal distribution, and Spearman correlation was used for variables displaying non-normal distributions. The level of significance was set at 5\%. For comparison among the affected and non-affected sides groups, the paired $t$-test was used for variables that showed normal distribution in the descriptive statistical methods (mean, standard deviation, minimum, and maximum) when comparing quantitative data. One-way analysis of variance (ANOVA) was performed to determine differences among the four types of Crowe classification. For comparison between these four groups, Tukey's range test was used among post hoc tests. The chi-squared test was used to evaluate differences in Dorr types.

\section{RESULTS}

\section{Bilateral Dysplasia of the Hip Group}

Twenty-five patients had hips with the same Crowe type and 21 with different Crowe types. The mean pelvic height was $204.7 \pm 15.4 \mathrm{~mm}$ (range, 164-240 mm), ischial height $47.5 \pm 8.2 \mathrm{~mm}$ (range, 28-65 mm), femur length was 425.1 $\pm 32.6 \mathrm{~mm}$ (range, $350-477 \mathrm{~mm}$ ), and tibial length was $345.9 \pm 29.5 \mathrm{~mm}$ (range, 259-395 $\mathrm{mm}$ ) in the group with the same Crowe type. The mean pelvic height was 217.3 $\pm 14.6 \mathrm{~mm}$ (range, 200-263 mm), ischial height 40.1 \pm $8.0 \mathrm{~mm}$ (range, 35-66 mm), femur length was $437.1 \pm$ $25.4 \mathrm{~mm}$ (range, 385-492 $\mathrm{mm}$ ), and tibial length was $351.0 \pm 22.6 \mathrm{~mm}$ (range, 318-397 $\mathrm{mm}$ ) in the group with different Crowe types. The relationship between Crowe types and pelvic, ischial, femoral and tibial lengths are summarized in Table 2. Overall, pelvic, ischial, femoral, and tibial heights were not related to the Crowe type in patients with bilateral DDH.

According to the Dorr classification, the 50 hips (25 patients) with bilateral DDH with the same Crowe type on the right side were type- $\mathrm{A}(\mathrm{n}=5)$, type- $\mathrm{B}(\mathrm{n}=18)$, and type-C $(n=2)$. Results for the left side were similar: type$A(n=7)$, type-B $(n=16)$, and type-C $(n=3)$. Therefore, there was no significant difference in Dorr types between the left and right femurs in patients with bilateral DDH $(P=0.712)$. 
Ömer Naci Ergin et al. Lower Extremity Morphology in Patients with Hip Dysplasia

A total of 42 hips (21 patients) with bilateral DDH but different Crowe types demonstrated results similar to those observed in the DDH group with the same Crowe types on each side: type-A $(n=6)$, type-B $(n=26)$, and type$\mathrm{C}(\mathrm{n}=10)$. It was determined that the Dorr types of femurs were not correlated with Crowe types of the hip in patients with bilateral DDH $(P=0.142)$.

\section{Unilateral Dysplasia of the Hip Group}

The mean pelvic height of patients with unilateral DDH was $209 \pm 2 \mathrm{~mm}$ on the affected side and $214 \pm 9 \mathrm{~mm}$ on the unaffected side. When compared to the pelvic height of the unaffected side, the pelvic height of the affected side was shorter in 15 patients, longer in 7 patients, and similar in 25 .

The mean ischial height of patients with unilateral DDH was $52.5 \pm 8.0 \mathrm{~mm}$ on the affected side and $54.1 \pm 9.0 \mathrm{~mm}$ on the unaffected side. The ischial height was shorter in the affected side in 9 patients, longer than the unaffected side in 3 , and similar in 39.

The mean femoral length of patients with unilateral DDH was $435.8 \pm 31.0 \mathrm{~mm}$ on the affected side and $439.4 \pm$ $29.0 \mathrm{~mm}$ on the unaffected side. The femoral length was shorter on the affected side in 19 patients, longer on the affected side in 15, and similar in 17.

The mean tibial length of patients with unilateral DDH was $353 \pm 28 \mathrm{~mm}$ on the affected side and $354 \pm 31 \mathrm{~mm}$ on the unaffected side. The tibial length was shorter on the affected side in 13 patients, longer on the affected side in 13, and similar in 25. Therefore, the Crowe type was not correlated with eventual lengthening in all heights.

According to the Dorr classification of patients with unilateral DDH, 10, 34, and 7 hips on the dysplastic side were type-A, type-B, and type-C, respectively, compared with 28,21 , and 2 type-A, type-B, and type-C hips, respectively on the normal side. The difference in the Dorr types of femurs was significant between dysplastic and normal sides in patients with unilateral DDH $(P=0.001)$.

\section{Coronal Alignment for all Dysplasia of the Hip}

The mean aLDFA was $80.5 \pm 3.9^{\circ}$, mLDFA was $86.1 \pm$ $3.7^{\circ}$, aMPFA was $86.7 \pm 13.7^{\circ}$, mLPFA was $87.8 \pm 13.4^{\circ}$, and MNSA was $132.4 \pm 14.2^{\circ}$ for all DDH hips. A coronal alignment analysis according to Crowe types is presented in Table 3. The only differences observed were in aMPFA and mLPFA between patients with Crowe types 2 and 3 $(P=0.030-0.028)$ (Table 4).

Table 2. The Relationship between Crowe Types and Pelvic, Ischial, Femoral, and Tibial Lengths

\begin{tabular}{lcccc}
\hline \hline & \multicolumn{2}{c}{ Same Crowe type $(\mathrm{n}=25)$} & \multicolumn{2}{c}{ Different Crowe types (n=21) } \\
\cline { 2 - 5 } & Same length & Different length & Same length & Different length \\
\hline Pelvic height (mm) & 14 & 11 & 11 & 10 \\
Ischial height (mm) & 13 & 12 & 12 & 9 \\
Femoral length (mm) & 7 & 18 & 4 & 17 \\
Tibial length (mm) & 13 & 12 & 9 & 12 \\
\hline
\end{tabular}

Table 3. Coronal Alignment Measurements for all DDH

\begin{tabular}{|c|c|c|c|c|c|c|c|c|c|}
\hline & \multicolumn{2}{|c|}{ Crowe 1 (n=77) } & \multicolumn{2}{|c|}{ Crowe $2(n=20)$} & \multicolumn{2}{|c|}{ Crowe 3 (n=18) } & \multicolumn{2}{|c|}{ Crowe 4 (n=28) } & \multirow{2}{*}{$P$-value } \\
\hline & Mean \pm SD & Min-Max & Mean \pm SD & Min-Max & Mean $\pm S D$ & Min-Max & Mean \pm SD & Min-Max & \\
\hline $\operatorname{aLDFA}\left({ }^{\circ}\right)$ & $80.8 \pm 3$ & 91 & $81.4 \pm 6$ & 74-99 & & & $77.5 \pm 3$ & $71-84$ & 0.32 \\
\hline $\operatorname{mLDFA}\left({ }^{\circ}\right)$ & $86.5 \pm 3$ & $75-95$ & $86.4 \pm 5$ & 78-101 & $85.3 \pm 2$ & $81-89$ & $83.4 \pm 2$ & $79-87$ & 0.71 \\
\hline aMPFA $\left.\left.\right|^{\circ}\right)$ & $86.4 \pm 9$ & $65-126$ & $93.1 \pm 18$ & $61-129$ & $84.7 \pm 12$ & $66-101$ & $83.2 \pm 16$ & $64-120$ & $0.03 *$ \\
\hline $\operatorname{mLPFA}\left({ }^{\circ}\right)$ & $88 \pm 12$ & $61-132$ & $82.4 \pm 12$ & $53-116$ & $93.2 \pm 12$ & $75-110$ & $88.9 \pm 17$ & $59-116$ & $0.02 *$ \\
\hline $\operatorname{MNSA}\left({ }^{\circ}\right)$ & $132.4 \pm 13$ & $81-164$ & $132.8 \pm 13$ & $110-155$ & $134.5 \pm 8$ & $124-150$ & $128.2 \pm 12$ & $100-160$ & 0.69 \\
\hline
\end{tabular}

DDH: developmental dysplasia of the hip, SD: standard deviation, Min: minimum, Max: maximum, aLDFA: anatomic lateral distal femoral angle, mLDFA: mechanical lateral distal femoral angle, aMPFA: anatomic medial proximal femoral angle, mLPFA: mechanical lateral proximal femoral angle, MNSA: femoral medial neck shaft angle.

$* P<0.05$. 
Hip\& Pelvis

Hip Pelvis 32(2): 85-92, 2020

Table 4. Coronal Alignment Measurements for Unilateral DDH

\begin{tabular}{lccccccc}
\hline \hline & \multicolumn{3}{c}{ Dysplastic hip } & \multicolumn{3}{c}{ Non-affected side } \\
\cline { 2 - 7 } & Mean & SD & Min-Max & Mean & SD & Min-Max & $P$-value \\
\hline aLDFA $\left({ }^{\circ}\right)$ & 81.34 & 3.48 & $76-91$ & 81.88 & 3.77 & $74-92$ & 0.381 \\
mLDFA $\left(^{\circ}\right)$ & 86.58 & 3.26 & $81-95$ & 88.96 & 2.98 & $84-96$ & $<0.001 *$ \\
aMPFA $\left({ }^{\circ}\right)$ & 86.14 & 8.21 & $70-114$ & 84.63 & 6.67 & $68-100$ & 0.462 \\
mLPFA $\left({ }^{\circ}\right)$ & 87.80 & 10.31 & $64-124$ & 88.44 & 8.38 & $74-130$ & 0.761 \\
MNSA $\left({ }^{\circ}\right)$ & 130.94 & 14.23 & $98-159$ & 129.26 & 5.58 & $109-145$ & 0.445 \\
\hline
\end{tabular}

DDH: developmental dysplasia of the hip, SD: standard deviation, Min: minimum, Max: maximum, aLDFA: anatomic lateral distal femoral angle, mLDFA: mechanical lateral distal femoral angle, aMPFA: anatomic medial proximal femoral angle, mLPFA: mechanical lateral proximal femoral angle, MNSA: femoral medial neck shaft angle.

$* P<0.05$.

\section{DISCUSSION}

Our initial hypothesis was that the Crowe type of DDH affects femoral alignment, femoral medullary canal, and lower extremity length. Our results indicate that in patients with unilateral DDH, the affected side may be shorter than the unaffected side regardless of the Crowe type. Deformity of the hip caused by developmental dysplasia produces anatomical changes in the proximal femur and pelvis, especially the acetabulum. In patients with bilateral DDH, femoral and pelvic lengths are unpredictable. In the femoral coronal alignment test, the average of all data vary widely, however, were within the normal limits.

When femoral length was evaluated in patients with acetabular dysplasia, Metcalfe et al. ${ }^{5)}$ noted that the affected femur was longer in the unilateral DDH group compared with the bilateral group who experienced more variability. Another study, conducted by Tamura et al. ${ }^{9}$ investigated femoral length in 90 patients with unilateral DDH. In their study, femurs were significantly longer in the DDH group compared with the control group. In that study, Tamura et al. ${ }^{9)}$ measured the femoral length which was defined as the vertical distance from the top of the greater trochanter to the most distal end of the intercondylar notch. The different points for measuring femoral length in this study may lead to differences between the lengths in those treated with unilateral DDH. In our study, the affected femur was also shorter in patients with unilateral DDH compared with the femur on the unaffected side. Kocabiyik et al. ${ }^{10}$ also found that the affected extremity was shorter in patients with unilateral DDH compared with the extremity on the unaffected side. A study conducted by Rai et al. ${ }^{11}$ revealed that the tibial length on the affected side in patients with DDH was $1 \mathrm{~cm}$ shorter compared with the unaffected side. In our series, the average tibial length on the affected side was also shorter in patients with unilateral DDH. Li et al. ${ }^{12)}$ also found that the unaffected side of the pelvis and ischial heights were higher in 100 patients with unilateral DDH as compared to the affected side. Similar to our series, the pelvis and ischial heights in the unilateral DDH group were shorter on the affected side, whereas data were variable in the bilateral group.

In tests of coronal alignment, aLDFA was within the normal range $\left(79-83^{\circ}\right)$ and valgus alignment was observed in $28(19.5 \%)$ of 143 hips, with mean values within normal limits. The varus alignment of twenty (13.9\%) hips were above the normal limit. In accordance with other studies, patients in this analysis with Crowe type 4 were found to have an average of $77.5^{\circ}$ valgus alignment ${ }^{13)}$. Furthermore, mLDFA values of patients with Crowe type 4 were below the normal range in this series. In a study conducted by Kocabiyik et al. ${ }^{10)}$, postoperative coronal alignment of 25 patients with Crowe type 4 was evaluated preoperatively and 1 year postoperatively. The mean mLDFA values in this study were within normal values $\left(85-90^{\circ}\right)$. In the same study, the mean values for aMPFA and mLPFA were found to be $98^{\circ}$ and $74^{\circ}$, respectively. Based on these values, the majority of hips in the series were determined to be coxa valga. In our series, aMPFA and MLPFA averages were determined to be $86^{\circ}$ and $87^{\circ}$, respectively, both within the normal ranges $\left(80-89^{\circ}\right.$ for aMPFA and $85-95^{\circ}$ for mLPFA). Mean values of aMPFA and mLPFA in 28 hips with Crowe type 4 in our series were within the normal range, and mLPFA was detected to be lower than $85^{\circ}$ in 39 hips (27.2\%). Again, the mean value of aMPFA was $\geq 89^{\circ}$ in 41 hips $(28.6 \%$ ), which is within the normal range.

To our knowledge, this is first study that evaluated hip morphology according to the Dorr classification in patients with DDH. In our series, patients with unilateral DDH were 
Ömer Naci Ergin et al. Lower Extremity Morphology in Patients with Hip Dysplasia

found to have significantly fewer Dorr type A femurs on the affected side compared to the healthy side. However, the difference between the hips of patients with bilateral DDH was not significant.

Although cementless prostheses are recommended for types A and B according to the Dorr classification, the recommendations for type-C remain controversial. However, some reports demonstrated that there is no difference in the cementless prosthesis survival among the three types ${ }^{14,15)}$.

There are limited studies evaluating pelvic parameters in patients with DDH. Albiñana et al. ${ }^{16)}$ studied a total of 83 patients with unilateral DDH to evaluate the relationship between the pelvic shape and innominate bone in the AP pelvic view. Statistically significant differences were observed in most cases, and pelvic asymmetry was obvious. In their opinion, changes of the pelvic shape occurred in patients with DDH, possibly due to growth disturbances in the triradiate cartilage. However, they did not follow subjects to adulthood to better understand pelvic asymmetry. Another study, Li et al. ${ }^{13)}$ observed that pelvic asymmetry exists in adult patients with DDH. In addition, the degree of asymmetry is correlated with the degree of hip dysplasia. In our study, we noted that the pelvic and ischial heights were higher in the unaffected hip of unilateral DDH patients. We suggest that pelvic asymmetry in adults with DDH may be due to pelvic deformation over years. Pelvic height should be evaluated to obtain better preoperative planning.

This study has some limitations. First, it is a retrospective study and a power analysis was not performed before beginning the study. Secondly, sagittal alignment and postoperative changes were not investigated. However, this is the first study on a large cohort that focused on changes in full-length lower extremity length and coronal alignment on patients with both unilateral and bilateral DDH who have undergone THA. Therefore, this study may be used as a reference for future studies to understand mechanical changes affecting the lower extremities in $\mathrm{DDH}$.

\section{CONCLUSION}

This study found that pelvic height and femoral length asymmetry are common and unpredictable in the presence of acetabular dysplasia. Femoral coronal alignment is also unpredictable. Surgeons operating on hips of patients with DDH should be aware of such possible anatomical variations. Especially in unilateral DDH patients, the pelvic height and femoral and tibial lengths on the affected side may be shorter compared with the unaffected side regardless of the Crowe type.

\section{CONFLICT OF INTEREST}

The authors declare that there is no potential conflict of interest relevant to this article.

\section{REFERENCES}

1. Harkess JW. Arthroplasty of the hip. In: Canale ST, Campbell WC, ed. Campbell's operative orthopaedics. 10th ed. St. Lousi (Mo): Mosby; 2002. 400-2.

2. Soukka A, Alaranta H, Tallroth K, Heliövaara M. Leg-length inequality in people of working age. The association between mild inequality and low-back pain is questionable. Spine (Phila Pa 1976). 1991;16:429-31.

3. Yildirim T, Guclu B, Karaguven D, Kaya A, Akan B, Cetin I. Cementless total hip arthroplasty in developmental dysplasia of the hip with end stage osteoarthritis: 2-7 years' clinical results. Hip Int. 2015;25:442-6.

4. Robertson DD, Essinger JR, Imura S, et al. Femoral deformity in adults with developmental hip dysplasia. Clin Orthop Relat Res. 1996;327:196-206.

5. Metcalfe JE, Banaszkiewicz P, Kapoor B, Richardson J, Jones CW, Kuiper J. Unexpected long femur in adults with acetabular dysplasia. Acta Orthop Belg. 2005;71:424-8.

6. Cameron HU, Botsford DJ, Park YS. Influence of the Crowe rating on the outcome of total hip arthroplasty in congenital hip dysplasia. J Arthroplasty. 1996; 11:582-7.

7. Renkawitz T, Weber T, Dullien S, et al. Leg length and offset differences above $5 \mathrm{~mm}$ after total hip arthroplasty are associated with altered gait kinematics. Gait Posture. 2016;49:196-201.

8. Dorr LD, Faugere MC, Mackel AM, Gruen TA, Bognar B, Malluche HH. Structural and cellular assessment of bone quality of proximal femur. Bone. 1993;14:231-42.

9. Tamura K, Takao M, Hamada H, Ando W, Sakai T, Sugano N. Femoral morphology asymmetry in hip dysplasia makes radiological leg length measurement inaccurate. Bone Joint J. 2019;101:297-302.

10. Kocabiyik A, Misir A, Kizkapan TB, et al. Changes in hip, knee, and ankle coronal alignments after total hip arthroplasty with transverse femoral shortening osteotomy for unilateral crowe type IV developmental dysplasia of the hip. J Arthroplasty. 2017;32:3449-56.

11. Rai NN, Siney PD, Fleming PA, Wroblewski BM. Discrepancy in the length of the tibiae in unilateral congenital dislocation of the hip. J Arthroplasty. 1999; 14:867-8.

12. Li YM, Li JH, Li B, Wang JX, Chen YS. The radiological research for pelvis asymmetry of unilateral developmental dysplasia of the hip in adult. Saudi Med J. 2016;37:13449.

13. Li Q, Kadhim M, Zhang L, Cheng X, Zhao Q, Li L. Knee joint changes in patients with neglected developmental hip dysplasia: a prospective case-control study. Knee. 2014; 21:1072-6. 


\section{Hip \& Pelvis}

Hip Pelvis 32(2): 85-92, 2020

14. Keisu KS, Orozco F, Sharkey PF, Hozack WJ, Rothman RH, McGuigan FX. Primary cementless total hip arthroplasty in octogenarians. Two to eleven-year follow-up. J Bone Joint Surg Am. 2001;83:359-63.

15. Berend KR, Lombardi AV, Mallory TH, Dodds KL, Adams JB. Cementless double-tapered total hip arthroplasty in patients 75 years of age and older. J Arthroplasty. 2004; 19:288-95.

16. Albiñana J, Morcuende JA, Delgado E, Weinstein SL. Radiologic pelvic asymmetry in unilateral late-diagnosed developmental dysplasia of the hip. J Pediatr Orthop. 1995; 15:753-62. 